\title{
Study of Luminous Emission from a Coaxial Plasma Discharge Device in the Presence of External Transverse Magnetic Field
}

\author{
Tarek M. Allam, Hanaa A. El-Sayed, Hanaa M. Soliman \\ Plasma Physics and Nuclear Fusion Department, Nuclear Research Center, AEA, Cairo, Egypt \\ E-mail:hanaa.elshamy@yahoo.com \\ Received January 8, 2011; revised February 8, 2011; accepted March 10, 2011
}

\begin{abstract}
The experimental investigations in this paper are focused on the study of luminous radiation emission from coaxial plasma discharge device and the effect of applied transverse magnetic field $B_{t r}$ on it. The experiment was done in $(1.5 \mathrm{KJ}-10 \mathrm{KV})$ coaxial plasma discharge device. The discharge is operated in Nitrogen gas at pressures from 1 to 2.2 torr. Helmholtz magnetic coils are placed outside the coaxial electrodes with its axis at a distance $=3 \mathrm{~cm}$ from the coaxial electrodes muzzle, then $B_{t r}$ with a maximum induction $\approx 0.85 \mathrm{~T}$ is applied perpendicularly to the expanded plasma from the coaxial electrodes muzzle. The diagnostics used in the measurements include a Rogowsky coil and a photomultiplier tube equipped with light collimator. The experimental results showed that the maximum intensity of luminous radiation is detected at axial distance (side view) $z=8 \mathrm{~cm}$ and gas pressure, $P=2.2$ torr. It also showed that the maximum value of axial luminous plasma zone velocity $=2.383 \times 10^{6} \mathrm{~cm} / \mathrm{s}$ at $z=11 \mathrm{~cm}$ and $P=1.4$ torr. In mode of presence of external $B_{t r}$, the investigations have shown that, at $P=1.4$ torr the maximum intensity of luminous radiation (detected at end-view position) is reduced by $17 \%$, the full width at half maximum, $F W H M$ of luminous radiation signal is increased by 40 times, while the luminous radiation signal is delayed by $t_{a}=438 \mu \mathrm{s}$. In two modes of operation $t_{a}$ and $F W H M$ have approximately a minimum values at $P=1.4$ torr.
\end{abstract}

Keywords: Coaxial Discharge, Luminous Radiation, Transverse Magnetic Field, Energy Losses

\section{Introduction}

Several studies were made in the past to detect the influence of applied external magnetic fields on the plasma behavior in coaxial plasma devices [1-5]. In coaxial electrodes discharge of $0.5 \mathrm{~kJ}$ [1] the angular velocity of plasma current sheath $P C S$ in the interelectrode discharge region was increased by addition of an externally excited axial magnetic field of $500 \mathrm{G}$ field along the coaxial electrodes. In coaxial plasma discharge of $4.5 \mathrm{~kJ}$ [2]. It has been observed that, with the application of external axial magnetic field along the coaxial electrodes of $3 \mathrm{kG}$, the $P C S$ was reduced from a complex multilayers structure to a single layer. If an externally excited axial magnetic field of $\left(10^{2}-10^{3} \mathrm{G}\right)$ was introduced at the end of the central electrode of $6 \mathrm{~kJ}$ coaxial discharge [3], the decay rate of $P C S$ was showed down. Study the effect of applied magnetic nozzle fields on coaxial plasma discharge showed that, the nozzle field will push the plasma through the field so that the plasma leaves the coaxial discharge without losing significant axial momentum [4]. Coaxial plasma discharge devices operating in a quasistationary mode, turbulences in plasma flow occur causing deformation of central electrodes. By using a ferromagnetic insert an additional magnetic field was generated in the coaxial electrodes. As a result, plasma turbulence and consequent wear of the anodes were strongly reduced [5].

A transverse magnetic field which was trapped ahead of the current sheath will reduce the ejection rate of plasma which occurred during the collapse stage of plasma focus discharge. This reduction should lead to a more uniform plasma of large dimensions [6]. The PCS at the coaxial electrodes muzzle had a conical shape with a thin luminous column on the axis. If transverse magnetic field of $0.1 \mathrm{w} / \mathrm{m}^{2}$ was applied between the coaxial electrodes at muzzle of the gun, the conical shape becomes more blunt and the central pinch becomes thicker due to the magnetic flux trapped in it [7]. A transverse magnetic field of $1 \mathrm{kG}$ applied at the coaxial muzzle impedes the motion of the $P C S$, and caused an increase in 
the soft X-ray emission intensity and the PCS become more stabilized $[2,8]$. Expanded PCS from the coaxial electrodes muzzle restricted by a transverse magnetic field of $280 \mathrm{G}$ applied directly after a coaxial muzzle, and the maximum velocity of expanded plasma was decreased by $33 \%$. Also the expanded plasma was contained by applied transverse magnetic field [9].

The purpose of the present paper is to examine the behavior of luminous radiation emission from a $P C S$ in axial phase (side view) and the effect of applied transverse magnetic field upon it (end view).

\section{Experimental Arrangement}

The coaxial plasma discharge device used in this paper consists of coaxial discharge chamber, energy storage system, the electrical power supply, the vacuum system and the gas flow inlet system [10].

A schematic diagram of the coaxial plasma discharge device is shown in, Figure 1. The device consists of two stainless steel electrodes, inner and outer electrodes, with diameters and lengths of $5 \mathrm{~cm}, 8.9 \mathrm{~cm}$ and $13 \mathrm{~cm}, 60 \mathrm{~cm}$ respectively. The inner and outer electrodes are insulated from each other by a tubular Perspex insulator of $1.5 \mathrm{~cm}$ length. A rectangular glass window of length $40 \mathrm{~cm}$ and width $0.4 \mathrm{~cm}$ parallel to the cylinder's axis is used for optical observation.

A capacitor bank of $30 \mu \mathrm{f}, 10 \mathrm{kV}$ is used to deliver a maximum discharge current of $\approx 68 \mathrm{kA}$ at charging voltage of $10 \mathrm{kV}$ from the power supply. The device is connected to the condenser bank via a spark gap switch and 12 coaxial cables. A high voltage pulse generator is used to trigger the spark gap switch, which in turn discharges the condenser bank. The inner electrode is negatively polarized with respect to the outer electrode. Figure 2 shows the electrical circuit of the coaxial plasma discharge device. The annular space between the two coaxial electrodes is admitted with nitrogen gas with pressure ranging from 1 to 2.2 torr after evacuation of discharge chamber to a suitable air pressure $\approx 10^{-3}$ torr.

A pair of Helmholtz coils each of 21 turns with outer diameter $24 \mathrm{~cm}$ and distance between centers is $12 \mathrm{~cm}$ is placed on each side of the outer electrode of coaxial system and a current of $2.413 \mathrm{KA}$ passes through the two coils to set a transverse magnetic field of $0.85 \mathrm{~T}$.

A photomultiplier tube with wavelength range (400 $\mathrm{nm}-700 \mathrm{~nm}$ ) is used in this work to give information about the luminous emission intensity from the coaxial plasma discharge. A slit of dark glass pipe (light collimator) is used to select a part of the illumination of the plasma sheath luminous zone and a cable of optical fiber is used to transmit the emitted light from the pipe to the photomultiplier slit. Figures $\mathbf{3}$ and $\mathbf{4}$ show the experimental arrangement of the array of photomultiplier and the discharge chamber in cases of side and end view respectively.

The data of experimental works were taken from an average of approximately from 5 to 7 shots for each gas pressures and axial distances under consideration.

\section{Experimental Results and Discussion}

The first part of this work deals with the behavior of luminous radiation emission from $P C S$ in axial direction along the coaxial electrodes (side view).

In this study the nitrogen gas pressure is the dominant parameter which affects the luminous radiation emission from plasma sheath and the other parameters of device under consideration are remain constant.

The maximum amplitude of the luminous radiation intensity, $I_{\text {rad }}$ as a function of axial distance, $Z$ and at different nitrogen gas pressure is shown in Figure 5. It can be seen from this figure that, the enhancement of $I_{r a d}$ is clear at $P=2.2$ torr, $z=8 \mathrm{~cm}$ from the coaxial electrodes breech, but $I_{\text {rad }}$ has a minimum value at the coaxial

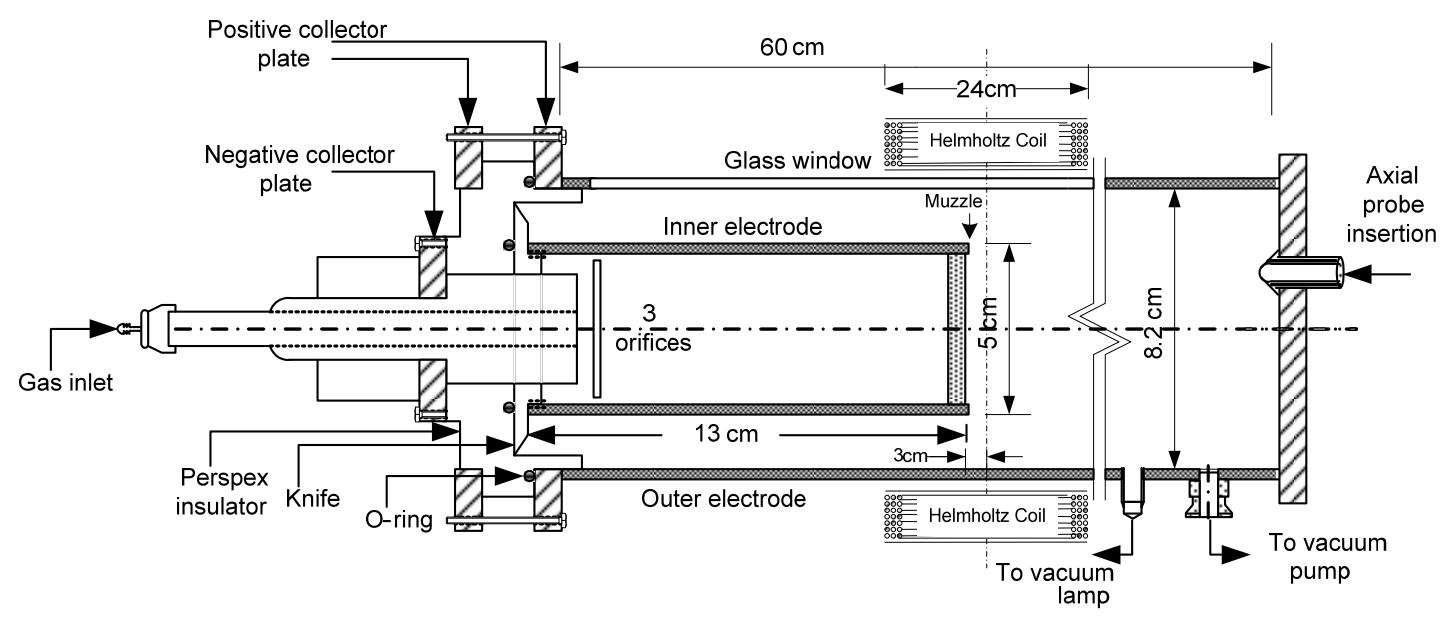

Figure 1. Coaxial plasma discharge device. 


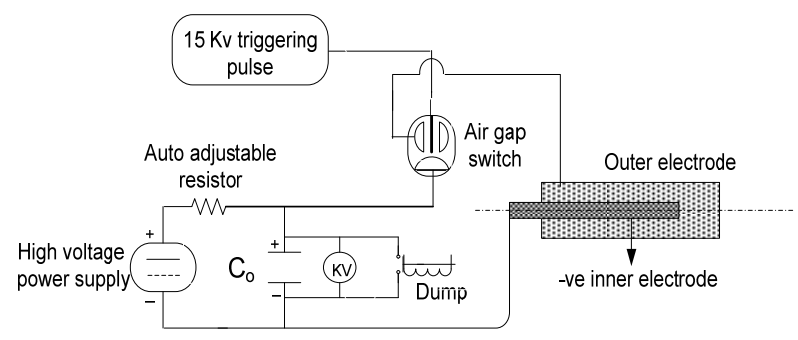

Figure 2. Electrical circuit of the coaxial plasma discharge device.

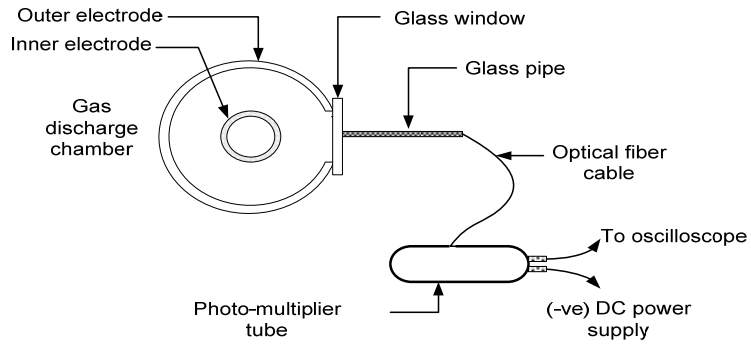

Figure 3. Experimental arrangement (side-view) of the array of photo-multiplier to the discharge chamber.

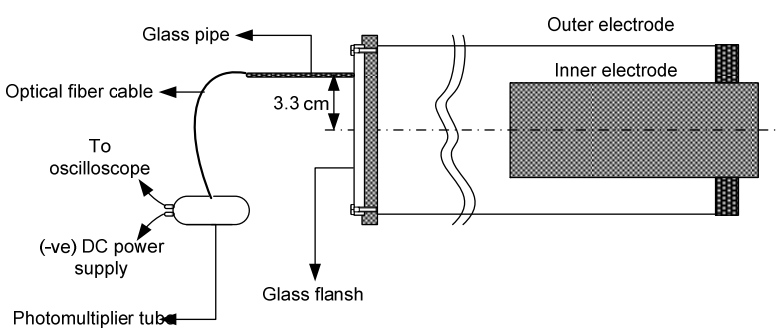

Figure 4. Experimental arrangement (end-view) of the array of photo-multiplier to the discharge chamber.

electrode muzzle, $z=12 \mathrm{~cm}$ and $P=2.2$ torr. Intensity of luminous radiation, $I_{\text {rad }}$ for all values of gas pressure and at $Z=12 \mathrm{~cm}$ is almost the same and it has approximately a minimum value, this may attributed to particle diffusion in freely ionized gas and three body recombination processes, also an increase of plasma temperature and velocity at this position. In general increased or decreased of $I_{\text {rad }}$ is related to different parameters such as plasma temperature and density as well as plasma velocity and energy losses from plasma.

From the arrival time of maximum amplitude of $\left(I_{\text {rad }}\right)$ data, the average luminous plasma zone velocity at the point of observation can be estimated during the axial phase. The variation of average luminous zone velocity $V_{L}$ as a function of axial distance $\mathrm{z}$ at a different gas pressures is illustrated in Figure 6. It indicates that $V_{L}$ gradually increases with increasing of axial distance, $\mathrm{z}$ to reach a maximum value at the coaxial muzzle for all values of gas pressures, except at $P=1$ torr and at axial distance from $5 \mathrm{~cm}$ to $9 \mathrm{~cm}$, this situation probably due

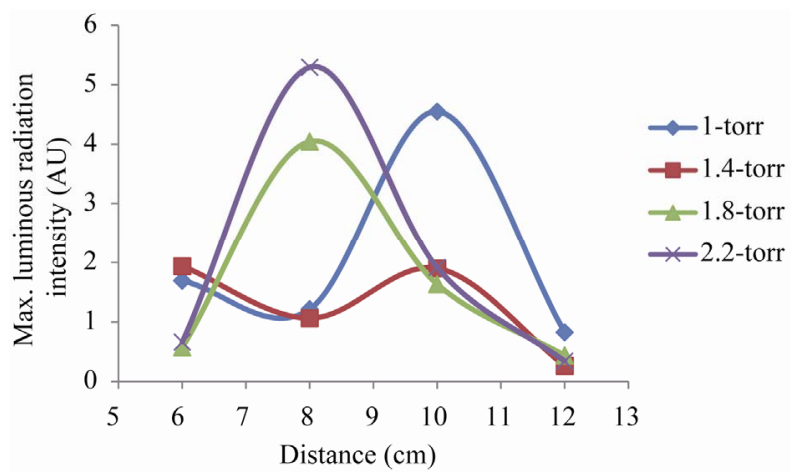

Figure 5. The maximum amplitude of the luminous radiation intensity as a function of axial distance at different gas pressures.

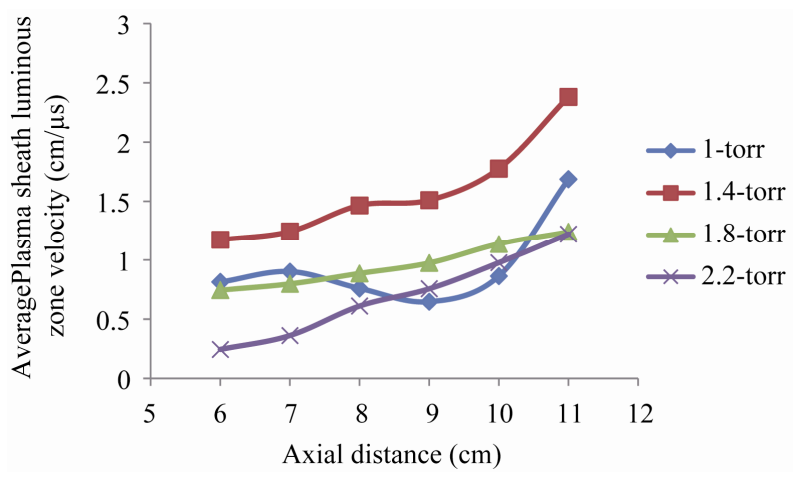

Figure 6. Axial distribution of plasma sheath luminous zone velocity.

to a some nonlinear phenomenon break the original behavior shown at $P=1.4,1.8,2.2$ torr, which need a further investigations. In general $V_{L(\max )}$ is detected at $P=$ 1.4 torr during the axial rundown phase, rather than $P=1$ torr, due to inefficient of Snowplough behavior [3] i.e. mass and current shedding effect $f_{m}, f_{c}$ respectively at $P=$ 1 torr play an important rule for $P C S$ motion.

Variation of $V_{L}$ and $I_{r a d}$ are plotted against the gas pressures and different $\mathrm{z}$ as shown in Figure 7, this results verified that, at a distance approaches to muzzle end the behavior of plasma sheath luminous zone velocity with a gas pressure has an opposite version with respect to the behavior of luminous zone intensity for most values of gas pressures. This behavior may due to, at axial distances closes to coaxial electrodes the average plasma sheath luminous zone velocity has a peak value for most values of gas pressures under consideration, this behavior may be due to less losses of plasma energy and increasing of plasma temperature, then a reduction of luminous radiation is occurred.

The second part of this study is concerned on the effect of applied external transverse magnetic field of maximum induction $B_{t r} \approx 0.85 \mathrm{~T}$ upon the luminous radiation emission from PCS (end-view). 

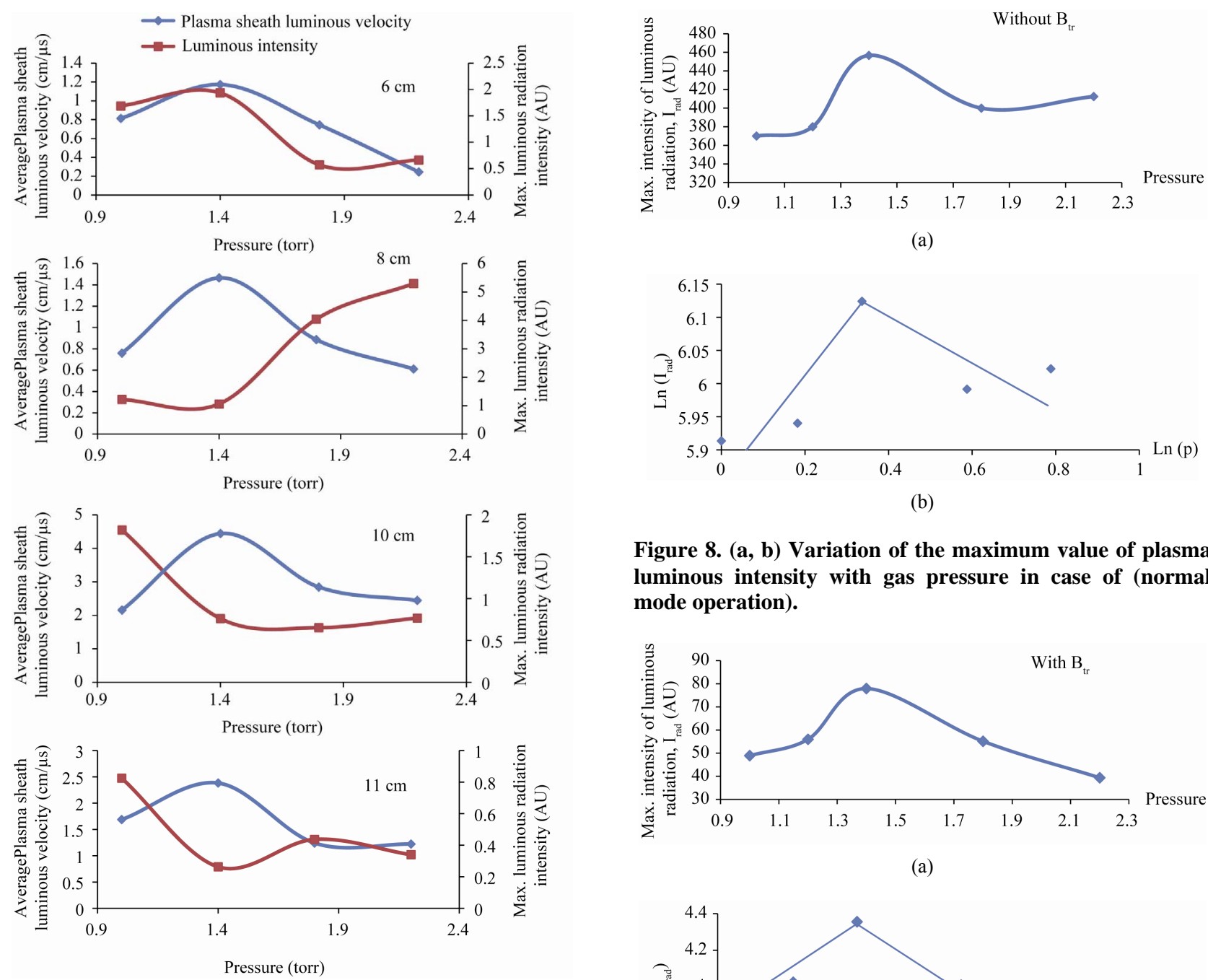

Figure 7. Variation of axial velocity and intensity of plasma luminous zone versus gas pressure.

A Helmholtz magnetic coils have been placed outside the outer electrode. The axis of them is located at $Z=3$ $\mathrm{cm}$ from coaxial electrodes muzzle to produce $B_{t r}$ across coaxial plasma discharge device and to detect the effect of $B_{t r}$ on plasma flow from coaxial electrodes muzzle.

In this experiment, the slit of the collimator is oriented at the end view of outer electrode axis at $Z=69 \mathrm{~cm}$ from the breech to view optically through a glass flansh the common end view of luminous radiation emission intensity from PS, this system is shown in Figure 4.

The effect of applied $B_{t r}$ on the maximum value of luminous radiation intensity as a function of gas pressure is illustrated in Figures 8 and 9, these figures demonstrated that in case of $B_{t r}=0, I_{\text {rad }}$ is increased with gas pressure to reach a maximum value at $P=1.4$ torr as $I_{\text {rad }} \alpha P^{0.79}$ and then it decreases as $I_{\text {rad }} \alpha P^{-0.34}$ but in case of applied $B_{\text {tr }}, I_{\text {rad }} \alpha P^{1.62}$ in the range from $P=1$ to 1.4 torr and $I_{\text {rad }}$ $\alpha P^{-1.5}$ in the range from 1.4 to 2.2 torr. 


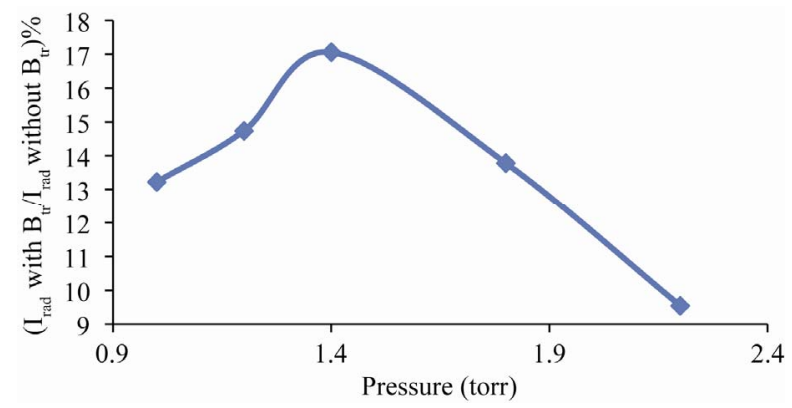

Figure 10. Percentage ratio of the maximum value of plasma luminous radiation intensity in cases of applied $B_{t r}$ and normal mode operation versus gas pressure.

pressure in two modes of operation is shown in Figure 11. These variations have approximately the same behaviors for all values of gas pressure except in the range from 1.2 to 1.4 torr the behavior has an opposite version. Also, from this figure, it must be noted that, $t_{a}$ (with $B_{t r}$ ) is much greater than the corresponding values in case of normal mode of operation, $B_{t r}=0$. Figure 12 shows the relation between the full width at half maximum (FWHM) of photomultiplier signal and the gas pressure in presence and absence of $B_{t r}$. It can be seen from this relation that, when $B_{t r}$ is applied ( $F W H M$ ) has a greater value than that in normal mode of operation. The minimum values of $t_{a}$ and $F W H M$ are detected approximately at $P$ $=1.4$ torr for two cases of operation while a maximum values of them are obtained at $P=1$ torr $\left(B_{t r}=0\right)$ and in the range from 1.8 to 2.2 torr $\left(B_{t r}=0.85 \mathrm{~T}\right)$ respectively. In general Figures 10-12 illustrate that, the plasma flow from the coaxial electrodes muzzle interacted with applied transverse magnetic field, then the plasma flow is contained and restricted by this magnetic field, also its motion is impeded [9] i.e. $t_{a}$ and FWHM in presence of $B_{t r}$ are greater than in normal mode operation, $B_{t r}=0$. Also a decrease in luminous radiation emission intensity in presence of $B_{t r}$ may due to particle diffusion and three body recombination processes, then presence of $B_{t r}$ causes a decrease in energy losses from plasma and plasma temperature raises may be expected.

\section{Conclusions}

The experimental work was done in a coaxial plasma discharge device with a nitrogen gas at pressure varying from 1 to 2.2 torr.

The study of the luminous radiation emerging from a $P C S$ during the axial phase is one of the most important features because it is the most efficient channel of energy losses from the plasma. In this study the luminous plasma radiations emission are visualized on two directions (side on and end on) of coaxial plasma device by using a photomultiplier with collimator system. The

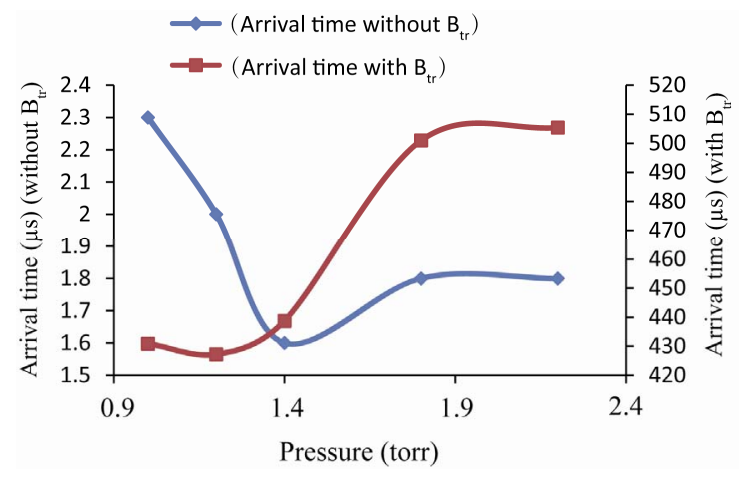

Figure 11. Arrival time in case of normal mode operation and case of applied external transverse magnetic field $B_{t r}$ versus gas pressure.

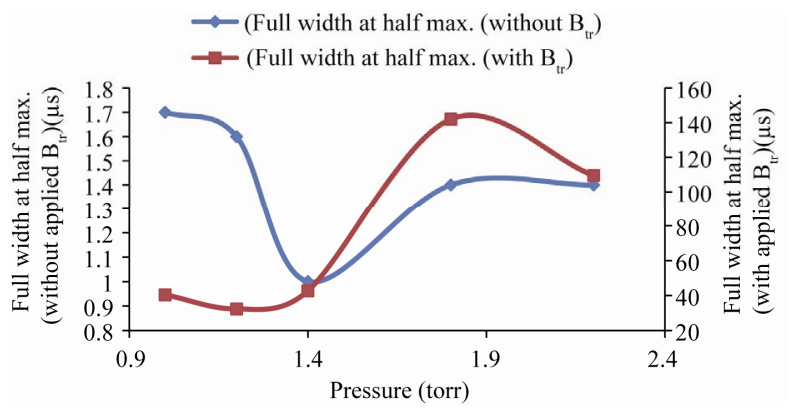

Figure 12. Full width at half maximum in cases of normal mode operation and applied external magnetic field versus gas pressure.

measurements were carried out in absence and presence of external applied transverse magnetic field of induction $\approx 0.85$ tesla.

The axial distribution of $I_{\mathrm{rad}}$ and $V_{L}$ were detected along the coaxial electrodes and at a different values of nitrogen gas pressures. The obtained experimental results reveal that, $I_{\text {rad }}$ has a peak value at a distance closes to coaxial electrodes muzzle, approximately at $8 \mathrm{~cm}$ and 10 $\mathrm{cm}$ for most values of gas pressures under consideration afterwards it decreases with increasing of axial distance to reach a minimum value at the muzzle, specially at $P=$ 2.2 torr. From the above results, one can concluded that, luminous radiation emission, $I_{\text {rad }}$ is due to excitation collision processes. Increasing of the luminous radiation intensity with axial distance can be contributed to the increase of plasma density when the PS scrapes the rest gas on its way along the coaxial electrodes while $I_{\text {rad }}$ decay at the muzzle is almost attributed to particle diffusion in fully ionized gases and three body recombination processes. In general an increase or decrease of $I_{\text {rad }}$ is related to different physical processes such as plasma temperature and density, plasma velocity and energy losses from plasma. Also experimental results illustrate that $V_{L}$ is increased gradually with increasing of axial distance for all values of gas pressure under considera- 
tion except at $P=1$ torr and at axial distance from $5 \mathrm{~cm}$ to $9 \mathrm{~cm}$, the behavior of $V_{L}$ versus $Z$ may probably due to some nonlinear phenomenon break the original behavior shown at $P=1.4,1.8$ and 2.2 torr, in future a more investigations will be carried out to study this behavior. Moreover the dependence of $I_{r a d}$ and $V_{L}$ on gas pressures and at different axial distance, $Z$ demonstrated that, at a distance near the end of a coaxial electrodes at $(Z=8 \mathrm{~cm}$ $11 \mathrm{~cm}), I_{\text {rad }}$ has a vise versa behavior with respect to $V_{L}$, this may due to an increase of axial $P C S$ force at axial distances mentioned previously, then an average luminous $P C S$ front velocity is increased and consequently a low intensity of $I_{\mathrm{rad}}$ is occurred during these distances as a result of less losses of plasma energy and increasing of plasma temperature.

Experimental results of variation of $t_{a}$ and $F W H M$ with a nitrogen gas pressures, illustrated that $t_{a}$ and FWHM (in presence of $B_{t r}$ ) are greater than the corresponding ones for $\left(B_{t r}=0\right)$. Also, a decrease of $I_{r a d}$ in presence of $B_{t r}$ is detected.

The conclusion obtained from the above results are as follows, applied $B_{t r}$ causes a reduction of luminous radiation emission intensity, $I_{r a d}$, delayed the arrival time, $t_{a}$ and increased of FWHM of PCS luminous zone signal i.e. $B_{t r}$ applied caused an impedes of the motion of PCS along the coaxial electrodes system for all values of experimental parameters under consideration. Then in general the energy losses from the plasma in the form of luminous radiation was decreased (with applied of $B_{t r}$ ) and an increase in plasma temperature may be expected.

\section{References}

[1] G. M. El-Aragi and E. El-Demardash, Proceedings of International Symposium, Research and Application of Plasmas, 2001.
[2] H. M. Soliman and M. M. Masoud, "Interaction of Plasma with Magnetic Fields in Coaxial Discharge," 18th ESP Conference on Controlled Fusion and Plasma Physics, Vol. 2, 1991, p. 139.

[3] W. H. Boskick, V. Nardi and W. J. Prior, "X-Ray Fine Structure of Dense Plasma in a Co-Axial Accelerator," Journal of Plasma Physics, Vol. 8, No. 1, 1972, pp. 7-20. doi:10.1017/S0022377800006899

[4] P. P. Hoyt, J. T. Schewer and K. E. Schoenberg "Magnetic Nozzle Design for Coaxial Plasma Accelerators," IEEE Transsections on Plasma Science, Vol. 23, No. 3, 1995, pp. 481-494.

[5] Z. Krzysztaf, "Reduction of Turbulence in an ImpulsePlasma Accelerator Operating in a Quasi-Stationary Mode," Vacuum, Vol. 42, No. 7, 1991, pp. 469-472.

[6] J. W. Mother, P. J. Battoms, P. J. Corpenter, A. H. Willoms and K. D. Ware, "Stability of the Dense Plasma Focus," Physics of Fluids, Vol. 12, No. 11, 1969, pp. 2343-2348. doi:10.1063/1.1692352

[7] L. Lindberg and C. T. Jacobsen, "Studies of Plasma Expelled from a Coaxial Plasma Gun," Physics of Fluids, Vol. 7, No. 11, 1968, pp. S44-S51. doi: $10.1063 / 1.1711090$

[8] H. M. Soliman and Masoud, "Correlation between Magnetic Tearing and X-Ray Emission in Coaxial Discharge," 17th EPS Conference on Controlled Fusion and Plasma Heating, Vol. 14B, No. 2, 1990, p. 630.

[9] H. M. Soliman, G. M. Elkashef and M. M. Masoud, "Coaxial Discharge and Transverse Magnetic Field," Contributions to Plasma Physics, Vol. 34, No. 4, 1994, pp. 587-591. doi:10.1002/ctpp.2150340406

[10] H. M. Hussien, T. M. Allam, H. A. El-Sayed and H. M. Soliman, "Characterization of 1.5 KJ Coaxial Plasma Discharge," Journal of Engineering and Applied Sciences, Vol. 56, No. 4, 2009, pp. 315-329. 